\title{
CYCLIC OPERATORS, COMMUTATORS, AND ABSOLUTELY CONTINUOUS MEASURES
}

\section{J. DOMBROWSKI}

\begin{abstract}
Commutator equations are used to study the relationship between the tridiagonal matrix structure of an unbounded cyclic selfadjoint operator and its spectrum. Sufficient conditions are given for absolute continuity. Results are related to the study of systems of orthogonal polyomials for which the measure of orthogonality is supported on an unbounded subset of the real line.
\end{abstract}

1. Introduction. Let $C$ be a selfadjoint operator with unit cyclic vector $\Phi$, defined on a separable Hilbert space $\mathscr{H}$. A basis $\left\{\Phi_{n}\right\}$ can be obtained for $\mathscr{H}$ by orthonormalizing $\left\{\Phi, C \Phi, C^{2} \Phi, \ldots\right\}$. It follows that the matrix with $(i, j)$ entry defined by $\left\langle C \Phi_{i}, \Phi_{j}\right\rangle$ has tridiagonal form:

$$
\left[\begin{array}{ccccc}
b_{1} & a_{1} & 0 & 0 & \cdots \\
a_{1} & b_{2} & a_{2} & 0 & \cdots \\
0 & a_{2} & b_{3} & 0 & \cdots \\
\vdots & \vdots & \vdots & \vdots &
\end{array}\right], \quad a_{n}>0, b_{n} \text { real. }
$$

This matrix, in turn, can be used to define an operator on the subset $\Omega$ of $l^{2}$ consisting of sequences for which matrix multiplication yields a sequence in $l^{2}$. If the given operator $C$ is bounded, then $\Omega=l^{2}$ and the matrix operator is unitarily equivalent to $C$. In fact, the set of bounded cyclic selfadjoint operators on $\mathscr{H}$ can be identified with the set of tridiagonal matrix operators satisfying (1.1) with $\left\{a_{n}\right\}$ and $\left\{b_{n}\right\}$ bounded. In this case the sequences $\left\{a_{n}\right\}$ and $\left\{b_{n}\right\}$ provide information about the spectral measure. Applications can be given to the study of systems of orthogonal polynomials for which the measure of orthogonality is supported on a bounded set (see [1-4]).

It is the purpose of this paper to consider matrices of the form (1.1) which represent unbounded cyclic selfadjoint operators defined on a dense subset of a separable Hilbert space $\mathscr{H}$. The tridiagonal structure of these matrices will be used to obtain information about the spectral measure of the corresponding selfadjoint operator. Applications will be given to the study of systems of orthogonal polynomials for which the interval of orthogonality is infinite.

Received by the editors January 30, 1986 and, in revised form, April 21, 1986.

1980 Mathematics Subject Classification (1985 Revision). Primary 47B15.

Key words and phrases. Absolute continuity, commutators, orthogonal polynomials. 
It will be shown below that (1.1) defines a selfadjoint operator $C$ if $\sum 1 / a_{n}=\infty$. In this case the spectral resolution $C=\int \lambda d E_{\lambda}$ gives rise to a spectral measure $\mu(\beta)=\left\|E(\beta) \phi_{1}\right\|^{2}$, defined for the Borel subsets of the real line. The polynomials defined by

$$
\begin{gathered}
P_{1}(\lambda)=1, \quad P_{2}(\lambda)=\frac{\lambda-b_{1}}{a_{1}}, \\
P_{n}(\lambda)=\frac{\left(\lambda-b_{n-1}\right) P_{n-1}(\lambda)-a_{n-2} P_{n-2}(\lambda)}{a_{n-1}}
\end{gathered}
$$

are orthonormal with respect to the usual inner product on $L^{2}(\mu)$ (see [6, Chapter VII]). These polynomials will be used to show that if $b_{n}=0, d_{n}=a_{n}-a_{n-1} \geqslant 0$, and $\sum_{n=1}^{\infty}\left|d_{n+1}-d_{n}\right|<\infty$ for all $n$, then $C$ has no eigenvalues. Under additional restrictions on the sequence $\left\{d_{n}-d_{n-1}\right\}$ it will be shown that $C$ is absolutely continuous, or equivalently, that $\mu$ is absolutely continuous.

From a theoretical point of view the techniques to be used are related to the work of C. R. Putnam on the use of commutator equations in the study of spectral measures (see [5], for example). The use of commutator equations is obviously complicated by the fact that the operators to be studied are unbounded and hence only densely defined.

2. Main results. The first lemma provides a sufficient condition for selfadjointness.

LEMMA 1. The matrix operator defined by (1.1) on the subset $\Omega$ of $l^{2}$ consisting of those sequence for which matrix multiplication yields a sequence in $l^{2}$, is selfadjoint if $\sum 1 / a_{n}=\infty$.

Proof. To show that the matrix operator $C$ is symmetric it is necessary to show that $\langle C x, y\rangle=\langle x, C y\rangle$ for any two sequences $x, y$ in $\Omega$. Toward this end let $x=\left\{x_{i}\right\}_{i=1}^{\infty}$ and $y=\left\{y_{i}\right\}_{i=1}^{\infty}$. It follows that

$$
\langle C x, y\rangle=\lim _{N \rightarrow \infty}\left[\left(b_{1} x_{1}+a_{1} x_{2}\right) \bar{y}_{1}+\sum_{i=2}^{N}\left(a_{i-1} x_{i-1}+b_{i} x_{i}+a_{i} x_{i+1}\right) \bar{y}_{i}\right]
$$

and

$$
\langle x, C y\rangle=\lim _{N \rightarrow \infty}\left[\left(b_{1} \bar{y}_{1}+a_{1} \bar{y}_{2}\right) x_{1}+\sum_{i=2}^{N}\left(a_{i-1} \bar{y}_{i-1}+b_{i} \bar{y}_{i}+a_{i} \bar{y}_{i+1}\right) x_{i}\right] .
$$

If $\alpha_{N}$ denotes the $N$ th partial sum of $\langle C x, y\rangle$ and $\beta_{N}$ denotes the $N$ th partial sum of $\langle x, C y\rangle$, then $\left|\alpha_{N}-\beta_{N}\right|=\left|a_{N} x_{N+1} \bar{y}_{N}-a_{n} x_{N} \bar{y}_{N+1}\right|$. Assume $\lim _{N \rightarrow \infty}\left|\alpha_{N}-\beta_{N}\right|$ $=2 d$, with $d>0$. Then for $N$ sufficiently large

$$
d \leqslant\left|\alpha_{N}-\beta_{N}\right| \leqslant\left|a_{N} x_{N+1} \bar{y}_{N}\right|+\left|a_{N} x_{N} \bar{y}_{N+1}\right| \text {. }
$$

It follows that $\left|x_{N+1} \bar{y}_{N}\right|+\left|x_{N} \bar{y}_{N+1}\right| \geqslant d / a_{N}$ for $N$ sufficiently large which contradicts the fact that $\sum_{N=1}^{\infty}\left[\left|x_{N+1} \bar{y}_{N}\right|+\left|x_{N} \bar{y}_{N+1}\right|\right]$ converges. Therefore $d=0$ and $C$ is symmetric. To show that $C$ is selfadjoint, assume that $y=\left\{y_{i}\right\}_{i=1}^{\infty}$ is in the domain of $C^{*}$ and that $C^{*} y=\omega=\left\{\omega_{i}\right\}$. Then $\left\langle C \Phi_{i}, y\right\rangle=\left\langle\Phi_{i}, C^{*} y\right\rangle$ implies that $\bar{\omega}_{i}=a_{i-1} \bar{y}_{i-1}+b_{i} \bar{y}_{i}+a_{i} \bar{y}_{i+1}$ and hence that $C^{*} y=C y$. Therefore $C$ is selfadjoint. 
The objective now is to analyze the spectral measure. Throughout the remainder of the paper the following will be assumed:

(i) $C$ is the cyclic selfadjoint operator defined on a dense subset of $l^{2}$ by the matrix (1.1) with $\sum 1 / a_{n}=\infty$.

(ii) The diagonal entries in (1.1) vanish.

(iii) The subdiagonal sequence $\left\{a_{n}\right\}$ monotonically increases to infinity.

Also, the following notation will be used:

(i) $\quad J=\left(T-T^{*}\right) / 2 i$ where $T \Phi_{n}=2 a_{n} \Phi_{n+1}$.

(ii) $J_{N}$ is the bounded operator obtained from $J$ by letting

$$
a_{n}=a_{N} \text { for } n \geqslant N \text {. }
$$

(iii) $C J_{N}-J_{N} C=-2 i K_{N}$ on $\Omega_{N}$ for $N=1,2,3, \ldots$

(iv) $d_{n}=a_{n}-a_{n-1}\left(a_{0}=0\right)$.

Note that for each $N, \Omega_{N}$ is a dense linear subset of $l^{2}$ which contains the basis vectors $\left\{\Phi_{n}\right\}$. Also $K_{N}$, defined by (2.2)(iii), is bounded if $\left\{d_{n}\right\}$ is a bounded sequence.

LEMMA 2. Assume (2.1) and (2.2). If $\left\{d_{n}\right\}$ is bounded and $C x=\lambda x$, then $\left\langle K_{N} x, x\right\rangle=0$ for $N=1,2, \ldots$

Proof. If $C x=\lambda x$ with $x \neq 0$, then $x$ is a nonzero multiple of $\left(P_{1}(\lambda), P_{2}(\lambda), P_{3}(\lambda), \ldots\right)$ with $\sum\left|P_{n}(\lambda)\right|^{2}<\infty$. Let $x_{j}=\left(P_{1}(\lambda), \ldots, P_{j}(\lambda), 0, \ldots\right)$. If $C_{\lambda}=C-\lambda I$ then $\left\langle C_{\lambda} J_{N} x_{j}, x\right\rangle-\left\langle J_{N} C_{\lambda} x_{j}, x\right\rangle=-2 i\left\langle K_{N} x_{j}, x\right\rangle$. Since $C_{\lambda} x=0$, $\left\langle J_{N} C_{\lambda} x_{j}, x\right\rangle=2 i\left\langle K_{N} x_{j}, x\right\rangle$. Thus since $K_{N}$ is a bounded operator, $\lim _{j \rightarrow \infty}\left|\left\langle C_{\lambda} x_{j}, J_{N} x\right\rangle\right|=2\left|\left\langle K_{N} x, x\right\rangle\right|$ exists. Assume $\lim \left|\left\langle C_{\lambda} x_{j}, J_{N} x\right\rangle\right|=2 p, p>0$. If $J_{N} x=\left(t_{1}, t_{2}, \ldots\right)$ then

$$
\begin{aligned}
\left|\left\langle C_{\lambda} x_{j}, J_{N} x\right\rangle\right| & =\left|t_{j} a_{j-1} P_{j-1}(\lambda)-\lambda t_{j} P_{j}(\lambda)+a_{j} P_{j}(\lambda) t_{j+1}\right| \\
& =\left|-t_{j} a_{j} P_{j+1}+a_{j} t_{j+1} P_{j}(\lambda)\right| .
\end{aligned}
$$

If $d_{n}=a_{n}-a_{n-1} \leqslant M$ then for $j$ sufficiently large

$$
p<\left|\left\langle C_{\lambda} x_{j}, J_{N} x\right\rangle\right| \leqslant 2 M j \max \left\{\left|t_{j} P_{j+1}(\lambda)\right|,\left|t_{j+1} P_{j}(\lambda)\right|\right\} \text {. }
$$

But this contradicts the fact that $\Sigma\left[\left|t_{j} P_{j+1}(\lambda)\right|+\left|t_{j+1} P_{j}(\lambda)\right|\right]<\infty$. It follows that $\lim _{j \rightarrow \infty}\left|\left\langle C_{\lambda} x_{j}, J_{N} x\right\rangle\right|=2\left|\left\langle K_{N} x, x\right\rangle\right|=0$.

ThEOREM 1. Assume (2.1). If $d_{n}=a_{n}-a_{n-1}$ and $\sum\left|d_{n+1}-d_{n}\right|<\infty$, then $C$ has no eigenvalues.

Proof. Suppose $\lambda$ is an eigenvalue with corresponding eigenvector $x=$ $\left(P_{1}(\lambda), P_{2}(\lambda), P_{3}(\lambda), \ldots\right)$. Choose $N_{0}$ sufficiently large such that for $n \geqslant N_{0}, d_{n}$ $<\frac{1}{2}\left(a_{n}-|\lambda / 2|\right)$ and $\sum_{n}^{\infty}\left|d_{i}-d_{i+1}\right|<\frac{1}{2}\left(a_{n}-|\lambda| / 2\right)$. Let $N$ be defined by $P_{N}^{2}(\lambda)=$ $\max _{n \geqslant N_{0}} P_{n}^{2}(\lambda)$. Then $C J_{N}-J_{N} C=-2 i K_{N}$ on a dense linear subset $\Omega_{N}$ of $\mathscr{H}$. Note that $K_{N}=\left[k_{i j}\right]$ where $k_{i i}=a_{i}^{2}-a_{i-1}^{2}$ for $i=1, \ldots, N, k_{i i}=a_{N}\left(a_{i}-a_{i-1}\right)$ 
for $i>N$, and $k_{i, i+2}=k_{i+2, i}=\frac{1}{2} a_{N}\left(a_{i+1}-a_{i}\right)$ for $i \geqslant N$. All other matrix entries of $K_{N}$ are zeros. It is shown in [2] that

$$
\sum_{n=1}^{N}\left(a_{n}^{2}-a_{n-1}^{2}\right) P_{n}^{2}(\lambda)=\left[a_{N-1} P_{N-1}(\lambda)-\frac{\lambda}{2} P_{N}(\lambda)\right]^{2}+\left(a_{N}^{2}-\frac{\lambda^{2}}{4}\right) P_{N}^{2}(\lambda) .
$$

Thus

$$
\begin{aligned}
\left\langle K_{N} x, x\right\rangle= & \sum_{1}^{N}\left(a_{i}^{2}-a_{i-1}^{2}\right) P_{i}^{2}(\lambda)+\sum_{N+1}^{\infty} a_{N} d_{i} P_{i}^{2}(\lambda)+\sum_{N+1}^{\infty} a_{N} d_{i} P_{i+1} P_{i-1} \\
\geqslant & {\left[a_{N-1} P_{N-1}(\lambda)-\frac{\lambda}{2} P_{N}(\lambda)\right]^{2}+\left(a_{N}^{2}-\frac{\lambda^{2}}{4}\right) P_{N}^{2}(\lambda)+\sum_{N+1}^{\infty} a_{N} d_{i} P_{i}^{2}(\lambda) } \\
& -a_{N} \sum_{N+1}^{\infty} \frac{1}{2} d_{i}\left[P_{i+1}^{2}+P_{i-1}^{2}\right] \\
\geqslant & {\left[a_{N-1} P_{N-1}(\lambda)-\frac{\lambda}{2} P_{N}(\lambda)\right]^{2}+\left(a_{N}^{2}-\frac{\lambda^{2}}{4}\right) P_{N}^{2}(\lambda)+\frac{1}{2} a_{N} d_{N+1} P_{N+1}^{2}(\lambda) } \\
& -\frac{1}{2} a_{N} d_{N} P_{N}^{2}(\lambda)-a_{N} P_{N}^{2}(\lambda) \sum_{N+1}^{\infty}\left|d_{i}-d_{i-1}\right| \\
> & {\left[a_{N-1} P_{N-1}(\lambda)-\frac{\lambda}{2} P_{N}(\lambda)\right]^{2}+\frac{1}{4} a_{N}\left(a_{N}-\frac{|\lambda|}{2}\right) P_{N}^{2}(\lambda) }
\end{aligned}
$$

If $P_{N}(\lambda)=P_{N-1}(\lambda)=0$, then $P_{N-2}(\lambda)=\cdots=P_{1}(\lambda)=0$ which cannot happen since $P_{1} \equiv 1$. Therefore $\left\langle K_{N} x, x\right\rangle \neq 0$ and by the previous lemma, $\lambda$ is not an eigenvalue.

Sufficient conditions will now be given for absolute continuity. Another lemma is needed. All notation remains as before.

Lemma 3. Assume (2.1) and (2.2). If the sequence $\left\{d_{n}\right\}$ is bounded, then for any bounded interval $\Delta$ and positive integer $N$,

$$
\left|\left\langle K_{N} E(\Delta) \Phi_{1}, E(\Delta) \Phi_{1}\right\rangle\right| \leqslant \frac{1}{2}\left\|J_{N}\right\||\Delta|\left\|E(\Delta) \Phi_{1}\right\|^{2}
$$

Proof. Let $\lambda$ be the midpoint of the bounded interval $\Delta$ and let $C_{\lambda}=C-\lambda I$. If $x_{n}=\sum_{i=1}^{n} \alpha_{i} \Phi_{i}$ where $\alpha_{i}=\left\langle E(\Delta) \Phi_{1}, \Phi_{i}\right\rangle$, then $C_{\lambda} J_{N} x_{n}-J_{N} C_{\lambda} x_{n}=-2 i K_{N} x_{n}$ and $\left\langle J_{N} x_{n}, C_{\lambda} E(\Delta) \Phi_{1}\right\rangle-\left\langle C_{\lambda} x_{n}, J_{N} E(\Delta) \Phi_{1}\right\rangle=-2 i\left\langle K_{N} x_{n}, E(\Delta) \Phi_{1}\right\rangle$. Since $J_{N}$ and $K_{N}$ are bounded, $\lim _{n \rightarrow \infty}\left\langle C_{\lambda} x_{n}, J_{N} E(\Delta) \Phi_{1}\right\rangle$ exists and it will be shown that this limit is $\left\langle C_{\lambda} E(\Delta) \Phi_{1}, J_{N} E(\Delta) \Phi_{1}\right\rangle$. If so then

$$
\begin{gathered}
\left\langle J_{N} E(\Delta) \Phi_{1}, C_{\lambda} E(\Delta) \Phi_{1}\right\rangle-\left\langle C_{\lambda} E(\Delta) \Phi_{1}, J_{N} E(\Delta) \Phi_{1}\right\rangle \\
=-2 i\left\langle K_{N} E(\Delta) \Phi_{1}, E(\Delta) \Phi_{1}\right\rangle
\end{gathered}
$$

and since $\left\|C_{\lambda} E(\Delta) \Phi_{1}\right\| \leqslant \frac{1}{2}|\Delta|\left\|E(\Delta) \Phi_{1}\right\|$, the lemma readily follows.

To evaluate the required limit, let $y_{n}=\sum_{i=1}^{n}\left\langle C_{\lambda} E(\Delta) \Phi_{1}, \Phi_{i}\right\rangle \Phi_{i}$. Since $E(\Delta) \Phi_{1}$ is in the domain of $C_{\lambda}$, the sequence $\left\{y_{n}\right\}$ converges to $C_{\lambda} E(\Delta) \Phi_{1}$, and so $\left\{J_{N} y_{n}\right\}$ also converges. Let $\omega_{n}=C_{\lambda} x_{n}-y_{n-1}=\left(a_{n-1} \alpha_{n-1}-\lambda \alpha_{n}\right) \Phi_{n}+a_{n} \alpha_{n} \Phi_{n+1}$. Then $\lim _{n \rightarrow \infty}\left\langle J_{N} \omega_{n}, E(\Delta) \Phi_{1}\right\rangle$ must exist. Since $E(\Delta) \phi_{1}=\sum_{i=1}^{\infty} \alpha_{i} \Phi_{i}$,

$$
\left|\left\langle\omega_{n}, J_{N} E(\Delta) \Phi_{1}\right\rangle\right|=\left|\left(a_{n-1} \alpha_{n-1}-\lambda \alpha_{n}\right) q_{n}+a_{n} \alpha_{n} q_{n+1}\right|
$$


where $q_{n}=\left\langle J_{N} E(\Delta) \Phi_{1}, \Phi_{n}\right\rangle$. Assume $\lim \left|\left\langle\omega_{n}, J_{N} E(\Delta) \Phi_{1}\right\rangle\right|=2 p$ where $p>0$. Since $\left\{d_{n}\right\}$ is bounded there exists $M$ such that $a_{n} \leqslant n M$. It follows that for $n$ sufficiently large

$$
p<\left|\left\langle\omega_{n}, J_{N} E(\Delta) \Phi_{1}\right\rangle\right|<n M\left[\left|\alpha_{n-1} q_{n}\right|+\left|\alpha_{n} q_{n}\right|+\left|\alpha_{n} q_{n+1}\right|\right] .
$$

But this contradicts the fact that $\sum\left[\left|\alpha_{n-1} q_{n}\right|+\left|\alpha_{n} q_{n}\right|+\left|\alpha_{n} q_{n+1}\right|\right]<\infty$. Therefore $\lim \left|\left\langle\omega_{n}, J_{N} E(\Delta) \Phi_{1}\right\rangle\right|=0$. Hence

$$
\lim _{n \rightarrow \infty}\left\langle C_{\lambda} x_{n}, J_{N} E(\Delta) \Phi_{1}\right\rangle=\left\langle C_{\lambda} E(\Delta) \Phi_{1}, J_{N} E(\Delta) \Phi_{1}\right\rangle
$$

as was to be shown.

Theorem 1 claims that if $\sum\left|d_{n+1}-d_{n}\right|<\infty$ then $C$ has no eigenvalues. The next theorem provides a sufficient condition for absolute continuity. Note that the conditions of the theorem are satisfied if $\left\{d_{n}\right\}$ is monotone increasing, bounded above, and $d_{n+1}-d_{n} \leqslant d_{n}-d_{n-1}$. In fact, all examples of this theorem are of this type.

Theorem 2. Assume (2.1). Let $d_{n}=a_{n}-a_{n-1}$. If $\left\{d_{n}\right\}$ is bounded and $d_{n+1}+$ $d_{n-1} \leqslant 2 d_{n}$ for $n \geqslant N \geqslant 2$ then $C$ is absolutely continuous.

Proof. The equation $C J_{N}-J_{N} C=-2 i K_{N}$ holds on a dense subset $\Omega_{N}$ where $J_{N}$ is defined in (2.2) and $K_{N}=\left[k_{i j}\right]$ with $k_{i i}=a_{i}^{2}-a_{i-1}^{2}$ for $i=1, \ldots, N, k_{i i}=$ $a_{N}\left(a_{i}-a_{i-1}\right)$ for $i>N$, and $k_{i, i+2}=\frac{1}{2} a_{N}\left(a_{i+1}-a_{i}\right)$ for $i \geqslant N$. All other matrix entries of $K_{N}$ are zeros. Note also that for any interval $\Delta$

$$
E(\Delta) \Phi_{1}=\sum_{i=1}^{\infty}\left\langle E(\Delta) \Phi_{1}, \Phi_{i}\right\rangle \Phi_{i}, \quad \text { where }\left\langle E(\Delta) \Phi_{1}, \Phi_{i}\right\rangle=\int_{\Delta} P_{i} d \mu .
$$

If $x_{n}=\sum_{i=1}^{n}\left\langle E(\Delta) \Phi_{1}, \Phi_{i}\right\rangle \Phi_{i}$ then for $n>N$

$$
\begin{aligned}
\left\langle K_{N} x_{n}, x_{n}\right\rangle= & \sum_{i=1}^{N}\left(a_{i}^{2}-a_{i-1}^{2}\right)\left|\int_{\Delta} P_{i} d \mu\right|^{2}+\sum_{i=N+1}^{n} a_{N}\left(a_{i}-a_{i-1}\right)\left|\int_{\Delta} P_{i} d \mu\right|^{2} \\
& +\sum_{i=N+1}^{n-1} a_{N}\left(a_{i}-a_{i-1}\right) \int_{\Delta} P_{i+1} \int_{\Delta} P_{i-1} \\
\geqslant & \sum_{i=1}^{N}\left(a_{i}^{2}-a_{i-1}^{2}\right)\left|\int_{\Delta} P_{i} d \mu\right|^{2}+\sum_{i=N+1}^{n} a_{N}\left(a_{i}-a_{i-1}\right)\left|\int_{\Delta} P_{i} d \mu\right|^{2} \\
& -\sum_{i=N+1}^{n-1} \frac{1}{2} a_{N}\left(a_{i}-a_{i-1}\right)\left[\left|\int P_{i+1}\right|^{2}+\left|\int P_{i-1}\right|^{2}\right] \\
\geqslant & a_{1}^{2}\left|\int_{\Delta} P_{1} d \mu\right|^{2} .
\end{aligned}
$$

This, together with the result of Lemma 3, implies that

$$
a_{1}^{2}\left\|E(\Delta) \Phi_{1}\right\|^{4} \leqslant \frac{1}{2}\left\|J_{N}\right\||\Delta|\left\|E(\Delta) \Phi_{1}\right\|^{2} .
$$


Let $\beta$ be a Borel subset of the real line of Lebesgue measure zero. Then for any $\varepsilon>0$ there exists a pairwise disjoint sequence of intervals $\left\{\Delta_{j}\right\}$ such that $\beta \subset \cup \Delta_{j}$ and $\Sigma\left|\Delta_{j}\right|<\varepsilon$. Since

$$
\mu(\beta) \leqslant \sum \mu\left(\Delta_{j}\right) \leqslant \frac{1}{2 a_{1}^{2}}\left\|J_{N}\right\| \sum\left|\Delta_{j}\right|
$$

it readily follows that $\mu(\beta)=0$.

Another sufficient condition for absolute continuity is provided by the following theorem. It will be shown in the next section that many examples can be constructed with the sequence $\left\{d_{n}\right\}$ monotonically decreasing to a nonnegative limit.

THEOREM 3. Assume (2.1). Let $d_{n}=a_{n}-a_{n-1}$. If $\left\{d_{n}\right\}$ is bounded and $d_{n+1}^{2} \leqslant$ $d_{n+2} d_{n}$ for $n \geqslant N \geqslant 2$ then $C$ is absolutely continuous.

Proof. The proof is similar to that of Theorem 2. With $J_{N}$ defined by (2.2) the operator $K_{N}$ is obtained from the equation $C J_{N}-J_{N} C=-2 i K_{N}$ which holds on a dense subset of $\mathscr{H}$. For any interval $\Delta$ let $x_{n}=\sum_{i=1}^{n}\left\langle E(\Delta) \Phi_{1}, \Phi_{i}\right\rangle \Phi_{i}$. Then for $n>N$,

$$
\begin{aligned}
\left\langle K_{N} x_{n}, x_{n}\right\rangle= & \sum_{i=1}^{N}\left(a_{i}^{2}-a_{i-1}^{2}\right)\left|\int_{\Delta} P_{i} d \mu\right|^{2}+\sum_{i=N+1}^{n} a_{N}\left(a_{i}-a_{i-1}\right)\left|\int_{\Delta} P_{i} d \mu\right|^{2} \\
& +\sum_{i=N+1}^{n-1} a_{N}\left(a_{i}-a_{i-1}\right) \int_{\Delta} P_{i+1} d \mu \int_{\Delta} P_{i-1} d \mu \\
\geqslant & \sum_{i=1}^{N}\left(a_{i}^{2}-a_{i-1}^{2}\right)\left|\int_{\Delta} P_{i} d \mu\right|^{2}+\sum_{i=N+1}^{n} a_{N}\left(a_{i}-a_{i-1}\right)\left|\int_{\Delta} P_{i} d \mu\right|^{2} \\
& -\left|\sum_{i=N+1}^{n-1} a_{N} d_{i} \int_{\Delta} P_{i+1} d \mu \int_{\Delta} P_{i-1} d \mu\right| .
\end{aligned}
$$

Since

$$
\begin{aligned}
\left|\sum_{i=N+1}^{n-1} a_{N} d_{i} \int_{\Delta} P_{i+1} d \mu \int_{\Delta} P_{i-1} d \mu\right| \\
\quad=a_{N} \sum_{i=N+1}^{n-1} \sqrt{d_{i-1}} \frac{d_{i}}{\sqrt{d_{i-1}}}\left|\int_{\Delta} P_{i+1} d \mu\right|\left|\int_{\Delta} P_{i-1} d \mu\right| \\
\leqslant \sum_{i=N+1}^{n-1} \frac{a_{N}}{2}\left[d_{i-1}\left|\int_{\Delta} P_{i-1}\right|^{2}+\frac{d_{i}^{2}}{d_{i-1}}\left|\int_{\Delta} P_{i+1} d \mu\right|^{2}\right]
\end{aligned}
$$

it follows that $\left\langle K_{N} x_{n}, x_{n}\right\rangle \geqslant a_{1}^{2}\left|\int P_{1} d \mu\right|^{2}$. The proof ends with the same argument used in the proof of Theorem 2.

3. Examples. The purpose of this final section is to illustrate the above results. Obviously all three theorems hold if the difference sequence $\left\{d_{n}\right\}$ is a constant sequence. As noted above, examples for Theorem 2 can be constructed by choosing 
$\left\{d_{n}\right\}$ to be monotone increasing, bounded above with $d_{n+1}-d_{n} \leqslant d_{n}-d_{n-1}$. Choose, for example, $d_{n}=\sum_{i=1}^{n} 1 / 2^{i}$. For this choice of $d_{n}$ the hypotheses of Theorem 2 are satisfied but those of Theorem 3 are not.

Theorem 3 requires that the sequence $\left\{d_{n}\right\}$ satisfy the condition $d_{n+1}^{2} \leqslant d_{n} d_{n+2}$ for $n \geqslant N$. This condition implies that if $d_{n} \leqslant d_{n+1}$ then $d_{n+1} \leqslant d_{n+2}$. It follows that $\left\{d_{n}\right\}_{n=N}^{\infty}$ is either monotone decreasing or eventually monotone increasing. If $\left\{d_{n}\right\}$ is monotone increasing for $n \geqslant M$ then $d_{M+k}<d_{M+1}\left(d_{M+1} / d_{M}\right)^{k-1}$ for $k=1,2, \ldots$, and so $\left\{d_{n}\right\}$ either diverges or becomes eventually constant. Since Theorem 3 also requires that $\left\{d_{n}\right\}$ be bounded it must be true that $\left\{d_{n}\right\}_{n=N}^{\infty}$ is montone decreasing.

Examples for Theorem 3 can be constructed by the following scheme. Choose $\frac{1}{2} \leqslant d_{2}<d_{1}$. If $\imath_{2}=d_{2}\left(d_{2} / d_{1}\right)$ then $r_{2}<d_{2}$ and it is possible to choose $d_{3}$ with $\max \left\{\imath_{2}, \frac{1}{3}\right\} \leqslant d_{3}<d_{2}$. If $d_{1}, \ldots, d_{n}$ have been chosen, let $\imath_{n}=d_{n}^{2} / d_{n-1}$ and choose $\max \left\{\imath_{n}, 1 /(n+1)\right\} \leqslant d_{n+1}<d_{n}$. The end result is a monotone decreasing sequence $\left\{d_{n}\right\}$ with $d_{n+1}^{2} \leqslant d_{n} d_{n+2}$ and $\sum d_{n}=\infty$. One specific example is $d_{n}=$ $1 / n$.

Another, perhaps more significant, example comes from the normalized Hermite polynomials which are orthonormal on $(-\infty, \infty)$ with respect to $d \mu=w(x) d x$ where $w(x)=e^{-x^{2}}$. These polynomials satisfy a recursion formula of the form (1.2) with $b_{n}=0$ and $a_{n}=\sqrt{n / 2}$. If $C$ is defined by (1.1) and $J$ by (2.2) then $C J-J C=-i I$ where $I$ denotes the identity opeator (see [5, pp. 63-64] for an interesting interpretation of the operators $C$ and $J)$. In this case $d_{n}=(1 / \sqrt{2})(\sqrt{n}-\sqrt{n-1})$ and it can be shown that $d_{n+1}^{2} \leqslant d_{n} d_{n+2}$ so that the conditions of Theorem 3 are satisfied. Since $\sqrt{n}-\sqrt{n-1}=1 /(\sqrt{n}+\sqrt{n-1})$ the required inequality is equivalent to $(\sqrt{n+1}+\sqrt{n})^{2} \geqslant(\sqrt{n}+\sqrt{n-1})(\sqrt{n+2}+\sqrt{n+1})$ which is easily verified.

In a related example, suppose $a_{n}^{2}-a_{n-1}^{2}=M$ for all $n$, so that $C J-J C=-2 i K$ where $K$ is a multiple of the identity. If $d_{n}=a_{n}-a_{n-1}$ then $d_{n+1}^{2} \leqslant d_{n} d_{n+2}$ is equivalent to $\left(a_{n+1}+a_{n}\right)^{2} \geqslant\left(a_{n}+a_{n-1}\right)\left(a_{n+2}+a_{n+1}\right)$ which follows from the observations that $a_{n-1} a_{n+1}=\left(a_{n}-d_{n}\right)\left(a_{n}+d_{n+1}\right) \leqslant a_{n}^{2}-d_{n+1}^{2} \leqslant a_{n}^{2}$ and that $a_{n-1} a_{n+2}=\left(a_{n}-d_{n}\right)\left(a_{n+1}+d_{n+2}\right) \leqslant a_{n} a_{n+1}$.

\section{REFERENCES}

1. J. Dombrowski, Tridiagonal matrix representations of cyclic selfadjoint operators, Pacific J. Math. 114 (1984), 325-334.

2. , Tridiagonal matrix representations of cyclic selfadjoint operators. II, Pacific J. Math. 120 (1985), 47-53.

3. A. Máté and P. Nevai, Orthogonal polynomials and absolutely continuous measures, Approximation Theory. IV (C. K. Chui, L. L. Schumaker, and J. D. Ward, eds.), Academic Press, New York, 1983, pp. 611-617.

4. P. Nevai, Orthogonal polynomials, Mem. Amer. Math. Soc., vol. 18, 1979, no. 213.

5. C. R. Putnam, Commutation properties of Hilbert space operators and related topics, Ergebnisse der Math., No. 36, Springer, 1967.

6. M. Stone, Linear transformations in Hilbert space, Amer. Math. Soc. Colloq. Publ., vol. 15, Providence, R. I., 1932; reprinted 1983.

Department of Mathematics and Statistics, Wright State University, Dayton, Ohio 45435 